\title{
Retinol transport proteins and concentrations in human amniotic fluid, placenta, and fetal and maternal sera
}

\author{
BY D. SKLAN \\ Faculty of Agriculture, Rehovot 76-100, Israel
}

\author{
AND I. SHALIT, N. LASEBNIK, Z. SPIRER AND Y. WEISMAN \\ Departments of Pediatrics, and Obstetrics and Gynaecology, Tel Aviv Medical Center, \\ Tel Aviv University, Sackler Medical School, Tel Aviv, Israel
}

(Received 10 October 1984 - Accepted 10 July 1985)

\begin{abstract}
1. The proteins binding retinol, and retinol concentrations, were determined in amniotic fluid, placental cytosol and in the fetal and maternal circulation.

2. In non-pregnant women,plasma retinol was almost exclusively found in a transthyretin-retinol-binding-protein complex whereas, in pregnant women, retinol-binding-protein-bound retinol was observed not complexed to transthyretin. This latter fraction increased in concentration with fetal age. These two fractions were the major retinol-protein complexes in amniotic fluid and their relative amounts changed with progress of gestation.

3. In fetal blood both of these fractions were again found, with higher proportions of retinol-bindingprotein-bound retinol in the umbilical artery than in the umbilical vein.
\end{abstract}

An adequate supply of retinol is necessary for normal fetal growth and developement but a deficiency or excess in the diet may be associated with teratogenic changes (Wilson et al. 1953; Nanda et al. 1977).

Retinol is usually transported in the blood bound to a specific retinol-binding protein (RBP) with a molecular weight (MW) of 19000-21000 daltons, this is in turn complexed to transthyretin (formerly prealbumin; TTR) which has a MW varying from 40000 to 60000 in different species (Smith \& Goodman, 1979).

Fetal metabolism of retinol and RBP has not been clearly delineated. In the rat fetus both RBP and retinol levels increased in parallel from 11 to $14 \mathrm{~d}$ of gestation, this was suggested to result from transplacental transport of retinol bound to RBP. Between 16 and $20 \mathrm{~d}$ of gestation, fetal levels of RBP increased greatly and this was attributed to fetal RBP synthesis; concomitantly, some fetal hepatic retinol storage began (Takahashi et al. 1977). From these results it was inferred that maternal RBP-retinol crosses the placenta and circulates in the fetus. In recent studies in humans, RBP has been detected in amniotic fiuid, and levels were correlated with retinol concentrations. It has also been suggested that retinol concentrations in amniotic fluid may be indicative of fetal malformation (Parkinson et al. 1982; Wallingford et al. 1983).

In studies in sheep it appeared that the fetal retinol transport in serum differs somewhat from maternal circulation (Donoghue et al. 1982).

Transport of retinol from mother to fetus is highly regulated (Moore, 1971; Bates, 1983) with little alterations in levels occurring in fetal or new-born animals despite large changes in maternal intake. However, the factors responsible for the control of fetal retinol levels have not been elucidated to date. In the present study we report on the vehicle of retinol transport in amniotic fluid, cord and maternal blood in the human, in an attempt to elucidate features of retinol transport to the fetus. 
Table 1. Retinol and retinol-binding-protein (RBP) concentrations in amniotic fluid from pregnant women at mid-term and term

(Mean 'values and standard deviations)

\begin{tabular}{|c|c|c|c|c|c|}
\hline & \multirow[b]{2}{*}{$n$} & \multicolumn{2}{|c|}{ Mid-term } & \multicolumn{2}{|c|}{ Term } \\
\hline & & Mean & SD & Mean & SD \\
\hline $\operatorname{Retinol}^{*}(\mu \mathrm{g} / \mathrm{l})$ & 34 & $25 \cdot 4 \dagger$ & $4 \cdot 2$ & $10 \cdot 4$ & $2 \cdot 8$ \\
\hline Retinol:protein (ng/mg) & 25 & $5 \cdot 4$ & $2 \cdot 5$ & $5 \cdot 7$ & 1.8 \\
\hline RBP (mg/l) & 10 & $6 \cdot 8$ & 0.8 & $5 \cdot 7$ & 0.9 \\
\hline RBP:protein $(\mu \mathrm{g} / \mathrm{mg})$ & 10 & $1 \cdot 4 \dagger$ & $0 \cdot 2$ & $3 \cdot 1$ & 0.7 \\
\hline
\end{tabular}

* Retinol was determined by high-pressure liquid chromatography and RBP by radial immunodiffusion.

$\dagger$ Mean value was significantly different from that at term $(P<0.05)$.

\section{MATERIALS AND METHODS}

Blood samples were obtained from pregnant women during pregnancy and at term, and from non-pregnant women including one group receiving oral contraceptives. Blood samples were also obtained from the umbilical artery and vein on delivery. Simultaneously, a sample was withdrawn from the maternal peripheral vein.

Placental samples were obtained from mothers following parturition, and were cut into small sections and soaked for 30-min intervals in six washes of saline $(9 \mathrm{~g}$ sodium chloride/1). Samples were homogenized with a high-speed homogenizer and cytosol prepared by centrifugation at $105000 \mathrm{~g}$ (Sklan et al. 1982).

Amniotic fluid samples were obtained from women at 16-18 weeks and 37-42 weeks of gestation. Mid-gestation samples were obtained by amniocentesis (for diagnosis of chromosome aberations); no aberations or metabolic defects were found in the fetuses used. Late gestation samples were obtained by artificial rupture of the amniotic fluid sac at term. Amniotic fluid samples were centrifuged at $3000 \mathrm{~g}$ for $10 \mathrm{~min}$ and the cell-free supernantant fraction taken for analyses. No amniotic fluid samples were visibly contaminated with blood.

Samples were either immediately analysed or frozen at $-18^{\circ}$ under nitrogen until analysis.

For retinol and retinyl ester determination, samples were extracted and subjected to high-pressure liquid chromatography (HPLC) on either a straight phase (Porasil; Waters Associates Inc., Milford, Mass., USA) or a reverse-phase (Partisil ODS 10; HPLC Technology, Macclesfield, UK) column as previously described (Sklan \& Halevy, 1984).

Separation of proteins was by gel-permeation chromatography with a TSK 3000 (LKB, Bromma) column eluting with 0.1 M-phosphate buffer containing $0.1 \mathrm{M}-\mathrm{NaCl}$ (Sklan \& Halevy, 1984). Additional gel filtrations on Sepharose 6B or Sephadex G-75 columns eluting with $0 \cdot 1 \mathrm{M}$-phosphate buffer, $\mathrm{pH} 7 \cdot 2$, containing $0 \cdot 1 \mathrm{M}-\mathrm{NaCl}$ were performed, in some cases samples were treated with $6 \mathrm{M}$-urea, and $3 \mathrm{M}$-urea was included in the eluting buffer (Sklan et al. 1982; Sklan \& Donoghue, 1982). Fractions eluting from the column were extracted with ethanol-petroleum spirit (b.p. $40-60^{\circ}$ ) and subjected to HPLC.

RBP levels were also determined by radial immunodiffusion using LC-Partigen plates (Behring Institute, Marburg).

Retinol fluorescence was determined as previously described using a Perkin-Elmer model 204 (Sklan \& Donoghue, 1982) and protein was determined by the dye-binding method of Bradford (1976). 


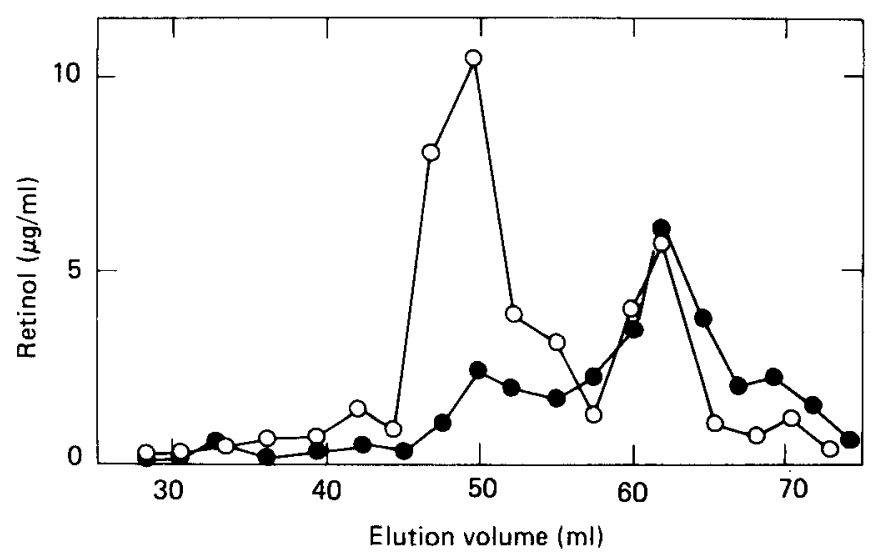

Fig. 1. Retinol elution profile of mid-term $\left(O^{\prime}\right)$ or term $(O)$ amniotic fluid from a Sepharose $6 \mathrm{~B}$ column $(480 \times 15 \mathrm{~mm})$ eluting with $50 \mathrm{~mm}$-Tris-hydrochloric acid, $\mathrm{pH} 7 \cdot 5$, containing $0.1 \mathrm{M}-\mathrm{HCl}$. The eluate was extracted and retinol determined by high-pressure liquid chromatography. The column was calibrated with blue dextran, thyroglobulin, apoferritin, phosphorylase B (EC 2.4.1.1), bovine serum albumin, ovalbumin, myoglobin, lysozyme and ribonuclease (EC 3.1.27.5).

Significance of differences was determined using Student's $t$ test.

Protocols were approved by the human subjects committee of Tel Aviv University.

\section{RESULTS}

Levels of retinol were determined in amniotic fluid both at mid-and full term. Retinol concentrations were higher on a per volume of amniotic fluid basis at mid-term but, when calculated on a protein basis, results were almost identical (Table 1). Retinyl esters were not detected.

Amniotic fluid was subjected to gel filtration on a Sepharose $6 \mathrm{~B}$ column, the eluate extracted and retinol determined by HPLC. The elution profile of retinol is shown in Fig. 1. In mid-term amniotic fluid, the major proportion of the retinol eluted very close to albumin, however, an additional major retinol-containing fraction was observed at a MW of approximately 20000 daltons. This peak comprised some $30-35 \%$ of mid-term amniotic fluid retinol. A smaller fraction between these two major fractions was also observed. Sepharose 6B gel filtration of amniotic fluid at term revealed retinol in the same two peaks as those at mid-term, although the distribution was different with the major retinol peak observed at a MW of 20000 . Both of these peaks were immunoreactive to anti-RBP. Gel filtration of the amniotic fluid was also carried out on Sephadex G-75 (Fig. 2) and the MW of the major retinol-binding peak at mid-term was found to be 64000-68000 daltons, and the lower MW peak 19000-21000 daltons. The intermediate peak had a MW of approximately 40000 daltons. These three peaks were immunoreactive with anti-RBP, and fluorescence determinations yielded an excitation spectrum with a maximum of $330 \mathrm{~nm}$ and an emission maximum of $470 \mathrm{~nm}$. Treatment of the isolated material from the $64000-66000$ MW peak with 6 M-urea followed by additional gel filtration resulted in appearance of retinol at MW 20000 only. Dialysis or increasing ionic strength changed somewhat the quantitative relation between these two peaks.

Determination of RBP either by immunodiffusion or by saturation of amniotic fluid with retinol and gel-permeation chromatography yielded similar results; values obtained by immunodiffusion are presented in Table 1. RBP was present in a molar excess (two- to 


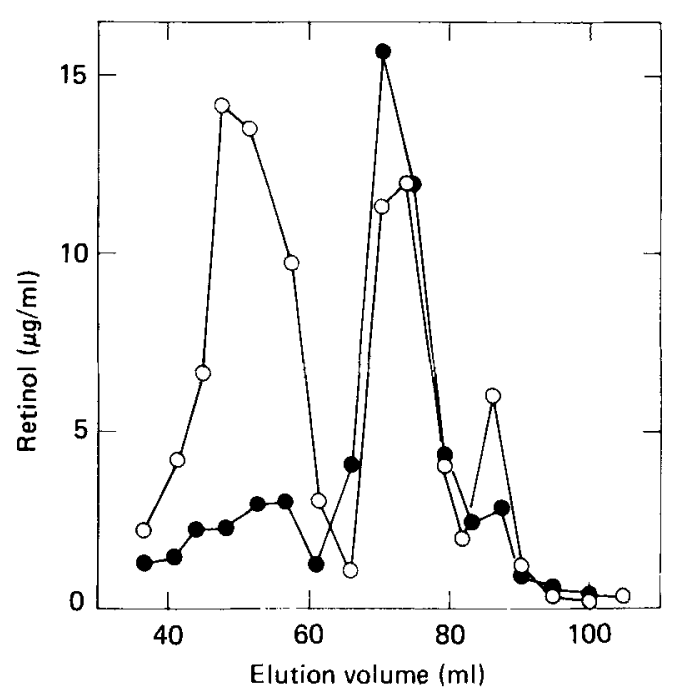

Fig. 2. Retinol elution profile of mid-term $(O)$ or term $(O)$ amniotic fluid from a Sephadex G-75 column $(600 \times 18 \mathrm{~mm})$ eluting with $50 \mathrm{~mm}$-Tris-hydrochloric acid, $\mathrm{pH} 7 \cdot 5$, containing $0 \cdot 1 \mathrm{M}-\mathrm{HCl}$. The column was calibrated with blue dextran, bovine serum albumin, carbonate dehydrase $(E C 4.2 .1 .1)$, myoglobin, lysozyme and ribonuclease (EC 3.1.27.5).

threefold) over retinol at mid-term, and this was more pronounced at term (seven- to eightfold). On a protein basis, significant differences were observed between mid-term and term RBP levels.

Cytosol and microsomes were prepared from placenta obtained at term and subjected to Sepharose 6B gel filtration (Fig. 3). Retinol was observed in peaks of MW of approximately $2 \times 10^{6}, 66000,20000$ and 15000 daltons. The 66000 and 20000 dalton peaks were immunoreactive to anti-RBP and, in the microsomes, a small immunoreaction was also observed. Similar gel filtration of maternal blood resulted in two retinol peaks, with the major retinol concentration found in the $64000-68000$ peak $(>80 \%$, Fig. 3). In non-pregnant women, with or without oral contraceptives, the $20000 \mathrm{MW}$ peak comprised less than $5 \%$ of protein-bound retinol (not shown). Retinol concentrations in serum of women ranged between 0.3 and $0.4 \mu \mathrm{g} / \mathrm{ml}$.

In fetal blood, retinol was found at concentrations of $0 \cdot 2-0.3 \mu \mathrm{g} / \mathrm{ml}$ and $\mathrm{RBP}$ at $25-35 \mu \mathrm{g} / \mathrm{ml}$; on gel filtration two retinol peaks were observed with MW of approximately 66000 and 20000 . Both of these peaks were immunoreactive with anti-RBP. However, the relative proportions of these two peaks differed in the umbilical vein and artery (Fig. 3), with a higher proportion of retinol observed in the $20000 \mathrm{MW}$ peak in the umbilical artery (almost $50 \%$ ). As described previously, urea treatment resulted in almost all the retinol appearing at 20000 daltons, and changing the ionic strength altered the distribution between these two peaks. Determination of the relative amounts of the 20000 dalton peak in women at different stages of pregnancy revealed increasing amounts of this peak as pregnancy progressed. The concentration ratio, $66000 \mathrm{MW}$ peak: $20000 \mathrm{MW}$ peak was correlated negatively with the week of pregnancy $(R-0.85, n 15)$. 


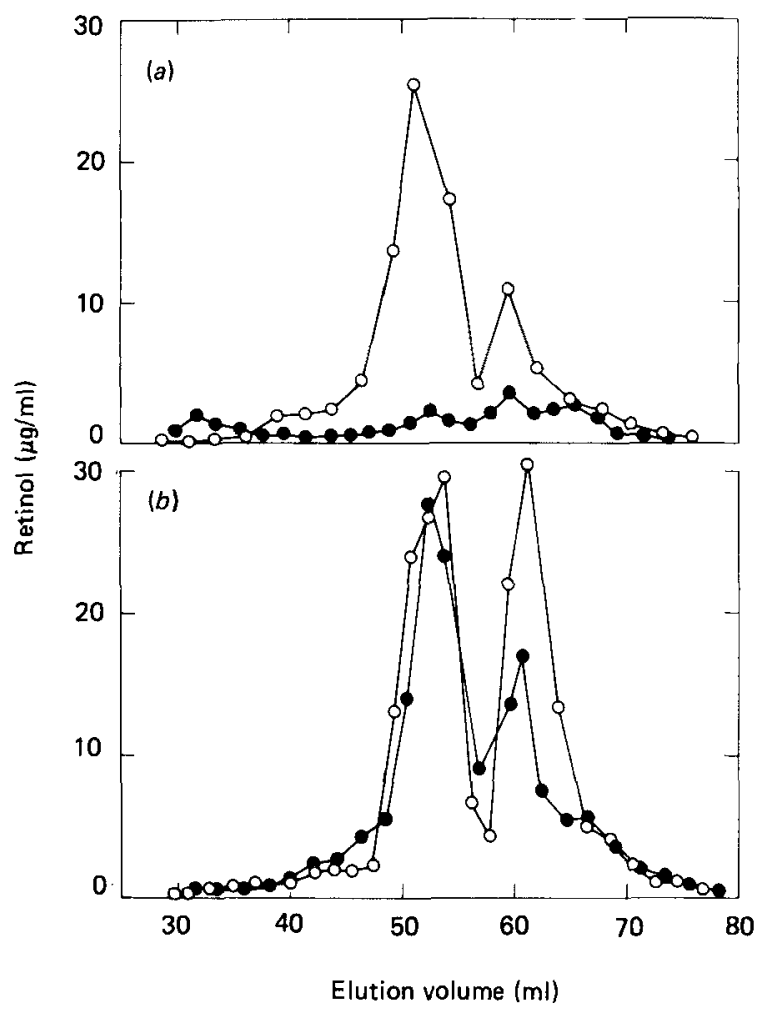

Fig. 3. Retinol elution profile of $(a)$ placental cytosol $(O)$, blood from mother $(O)$ and $(b)$ umbilical artery $(O)$ and umbilical vein $(O)$ from a Sepharose $6 \mathrm{~B}$ column $(480 \times 15 \mathrm{~mm})$ eluting with $50 \mathrm{~mm}$ Tris-hydrochloric acid, $\mathrm{pH} 7 \cdot 5$, containing $0 \cdot 1 \mathrm{M}$-sodium chloride. The column was calibrated with blue dextran, bovine serum albumin, carbonate dehydrase ( $E C$ 4.2.1.1), myoglobin, lysozyme and ribonuclease (EC 3.1 .27 .5$)$.

\section{DISCUSSION}

Retinol in amniotic fluid and fetal circulation appears to be bound both to RBP and to RBP further complexed to TTR. This is in contrast to the single retinol-RBP-TTR peak observed in non-pregnant circulation. This was deduced from the MW of fractions observed, the behaviour towards dissociating materials and the immunoreactivity to anti-RBP. These peaks of RBP and RBP-TTR co-elute in fetal serum and in amniotic fluid. Retinol distribution in the placental cytosol exhibited typical intracellular RBP of MW $2 \times 10^{6}$ and 14000-16000 daltons (Sklan et al. 1982). The latter may include the fetal intracellular RBP described by Ong (1984). Additional RBP fractions in placental cytosol co-eluted with those found in the amniotic fluid. This indicates that fractions found in the amniotic fluid and fetal circulation may originate in the placenta.

In sera of non-pregnant women, retinol was almost exclusively TTR-RBP bound; however, in pregnant women, increasing proportions of the serum retinol were complexed to $\mathrm{RBP}$ alone. In fetal serum a large proportion of the retinol-RBP was also not complexed to TTR, and this was especially apparent in the umbilical artery. In human fetal blood, retinol has been previously reported to be mainly bound to what appears to be TTR-RBP, although this was after ion-exchange chromatography with a $\mathrm{NaCl}$ gradient which influences RBP-TTR binding (Ismadi \& Olson, 1975). In fetal sheep blood, however, a considerable proportion of the serum retinol was not TTR-RBP bound but was bound to 
proteins with MW between that of the TTR complex and RBP (Donoghue et al. 1982). Maternal and fetal RBP appear to be identical on the basis of fluorescence, electrophoretic, chromatographic (Ismadi \& Olson, 1975) and, as shown in the present study, immunoreactive behaviour. However, the concentration or properties of TTR may be different in the fetus and mother.

Retinol and RBP levels in amniotic fluid and maternal and fetal plasma found here were similar to those described in previous studies (Vahlquist et al. 1975; Wallingford et al. 1983).

Transfer of proteins across the placenta apears to be inversely dependent on the molecular size (Burnett \& Bradwell, 1980), although some transfer of molecules of over 100000 daltons has also been reported (Wild, 1974; Hemmings \& Hemmings, 1979). Retinol transport across the placenta has been assumed to be effected by RBP (Takahashi et al. 1977); however, placental transfer may not be connected with the formation of the complex between RBP and TTR. One possible explanation of non-TTR-bound RBP in the fetal circulation and amniotic fluid could be that during transplacental transfer the TTR-RBP complex is partially modified, probably in the TTR moiety. The surviving complex may either circulate as such, or dissociate to RBP. Another possibility would be that transport of maternal retinol-RBP across the placenta occurs after dissociation of TTR from the TTR-RBP complex at the surface of or within the placenta. This could result in the appearance of RBP not complexed to TTR in the fetus. A further possibility would be transfer of retinol not bound to proteins, across the placenta; a fetal RBP - retinol complex would then occur and could further bind to other entities including fetal TTR. Only small amounts of RBP were detected in placental microsomes, thus indicating that placental synthesis of RBP probably did not occur on a large scale. The non-TTR-complexed RBP in the circulation of pregnant mothers may be explained in the light of recent studies indicating fetal to maternal retinol transfer (Donoghue et al. 1982; Ismadi \& Olson, 1982). If this fetal to maternal transport also does not involve the TTR-RBP complex this may provide the source of the maternal non-TTR-complexed RBP.

In mid-term amniotic fluid a molar excess of RBP over retinol was observed both in the present study and in a previous study (Wallingford et al. 1983). This excess could represent apo-RBP following delivery of retinol to tissues or, alternatively, arise from fetal apo-RBP.

Levels of RBP are relatively constant in maternal serum despite wide variations in retinol intake (Smith \& Goodman, 1979); in excessive retinol intakes, plasma retinyl ester concentration increases, but little change in RBP occurs (Mallia et al. 1975). In addition, these variations in maternal retinol intake do not result in parallel changes in fetal (or new-born) retinol levels (Moore, 1971; Bates, 1983). This could be explained if fetal retinol uptake is mediated by circulating maternal RBP.

\section{REFERENCES}

Bates, C. J. (1983). Proceedings of the Nutrition Society 42, 65-79.

Bradford M. M. (1976). Analytical Biochemistry 72, 248-252.

Burnett, D. \& Bradwell, A. R. (1980). Biology of the Neoate 37, 302-307.

Donoghue, S., Richardson, D. W., Sklan, D. \& Kronfeld, D. S. (1982). Journal of Nutrition 112, 2197 -2203.

Hemmings, C. \& Hemmings, W. A. (1979). In Protein Transmission Through Living Membranes, pp. 269-276

[W. A. Hemmings, editor]. Amsterdam: Elsevier.

Ismadi, S. D. \& Olson, J. A. (1975). American Joumal of Clinical Nutrition 28, 967-972.

Ismadi, S. D. \& Olson, J. A. (1982). International Journal of Vitamin and Nutrition Research 52, 111-118.

Mallia, A. K., Smith, J. E. \& Goodman, D. S. (1975). Journal of Lipid Research 16, 180-188.

Moore, T. (1971). International Journal of Vitamin and Nutrition Research 41, 301-306.

Nanda, R., May, D. L. \& Lite, S. (1977). Oral Biology 22, 613-618.

Ong, D. (1984). Journal of Biological Chemistry 259, 1476-1482.

Parkinson, C. E., Tan, J. C. Y. \& Gal, I. (1982). British Journal of Obstetrics and Gynaecology 89, 935-939. 
Sklan, D., Blaner, W. S., Adachi, N., Smith, J. E. \& Goodman, D. S. (1982). Archives of Biochemistry and Biophysics 214, 35-44.

Sklan, D. \& Donoghue, S. (1982). Biochimica et Biophysica Acta 711, 532-538.

Sklan, D. \& Halevy, O. (1984). British Journal of Nutrition 52, 107-114.

Srith, J. E. \& Goodman, D. S. (1979). Federation Proceedings 38, 60-65.

Takahashi, Y. I., Smith, J. E. \& Goodman, D. S. (1977). American Journal of Physiology 223, E263-E272.

Vahlquist, A., Rask, L., Peterson, P. A. \& Berg, T. (1975). Scandinavian Journal of Laboratory and Clinical Investigation 35, 569-575.

Wallingford, J. C., Milunsky, A. \& Underwood, B. A. (1983). American Journal of Clinical Nutrition 38, 377-381.

Wild, A. E. (1974). Symposium of the Society of Experimental Biology 28, 521-546.

Wilson, J. G., Roth, C. B. \& Warkany, J. (1953). American Journal of Anatomy 92, 189-217. 